\title{
Revista Colombiana de

\section{Capítulo 10. Indicadores de calidad en la atención de pacientes con falla cardiaca}

\section{Adriana Torres}

\author{
Departamento de Cardiología, Fundación Cardioinfantil-Instituto de Cardiología, Bogotá, Colombia
}

Recibido el 16 de noviembre de 2015; aceptado el 12 de enero de 2016

Disponible en Internet el 1 de febrero de 2016

\section{PALABRAS CLAVE Insuficiencia cardiaca; Pronóstico; Estudios de seguimiento}

\section{KEYWORDS}

Cardiac failure; Prognosis;

Follow-up studies
Resumen La implementación de estrategias de atención como algoritmos, listas de chequeo e indicadores pretende reducir las variaciones en la práctica y mejorar los resultados de la atención en salud.

Objetivo: Presentar los indicadores más importantes de calidad que deben implementarse en la atención de los pacientes con falla cardiaca para lograr programas de excelencia.

Metodología: Se realizó una revisión de la literatura disponible.

Conclusión: La calidad de la atención en las Unidades de Falla Cardiaca depende de la capacitación de su personal, de atender el número de pacientes suficientes para mantener las competencias del equipo, de su estructura multidisciplinaria y del registro de indicadores al alta hospitalaria y del seguimiento de los pacientes.

(c) 2016 Publicado por Elsevier España, S.L.U. en nombre de Sociedad Colombiana de Cardiología y Cirugía Cardiovascular. Este es un artículo Open Access bajo la licencia CC BY-NC-ND (http://creativecommons.org/licenses/by-nc-nd/4.0/).

\section{Chapter 10. Quality indicators in the care of patients with heart failure}

Abstract Care strategies such as algorithms, checklists and indicators are implemented in order to reduce variations in practice and to improve healthcare results.

Objective: To present the most important quality indicators to implement in the care of heart failure patients to achieve programmes of excellence.

Methodology: A review of the available literature was undertaken.

Conclusion: The quality of care in heart failure units depends on the training of their personnel, on attending a sufficient number of patients in order to maintain the team's skills, on their multidisciplinary structure and on the indicator registry on hospital discharge, and on patient follow-up.

(C) 2016 Published by Elsevier España, S.L.U. on behalf of Sociedad Colombiana de Cardiología y Cirugía Cardiovascular. This is an open access article under the CC BY-NC-ND license (http://creativecommons.org/licenses/by-nc-nd/4.0/).

Correo electrónico: torrnav@hotmail.com 


\section{Introducción}

La implementación de estrategias de atención como algoritmos, listas de chequeo e indicadores pretende reducir las variaciones en la práctica y mejorar los resultados de la atención en salud ${ }^{1-3}$. Por tanto, la utilización óptima de recursos es primordial y para ello es importante determinar qué componentes de una intervención son efectivos a largo plazo con el fin de lograr programas ajustados a nuestra realidad y sostenibles ${ }^{4,5}$. Previamente se vieron los componentes de una unidad de falla cardiaca; sin embargo, el continuo de la atención inicia en la hospitalización en la cual también debe haber unas características que determinan la calidad de la atención (tabla 1).

\section{Elementos de la unidad}

La calidad de la atención en las Unidades de Falla Cardiaca depende igualmente de la capacitación de su personal y de atender el número de pacientes suficientes para mantener las competencias del equipo $0^{6,7}$. De esta forma, un
Tabla 1 Medidas de calidad al alta hospitalaria de un paciente con falla cardiaca

\begin{tabular}{ll}
\hline Ítem & \\
\hline Instrucciones al alta & - Cita de control \\
Educación & - Teléfono de contacto \\
& hidrosalina \\
& - Medicación \\
& - Monitorización de peso \\
& $\begin{array}{l}\text { contactar } \\
\text { Cignos de alarma y cómo }\end{array}$ \\
Medición de la fracción de & $\begin{array}{l}\text { Debe consignarse en el } \\
\text { resumen de alta }\end{array}$ \\
eyección & En caso de no uso justificar la \\
Uso de IECA o ARA II & razón \\
Clase funcional/Nivel de & Utilizar clasificación de la \\
actividad física & NYHA \\
\hline
\end{tabular}

Tabla 2 Indicadores de calidad

\begin{tabular}{|c|c|c|c|}
\hline Tipo & Nombre & Numerador & Denominador \\
\hline Cobertura & Cobertura & $\begin{array}{l}\text { Número de pacientes incluidos en } \\
\text { el programa }\end{array}$ & $\begin{array}{l}\text { Número de pacientes identificados } \\
\text { para ingresar al programa }\end{array}$ \\
\hline Continuidad & Continuidad & $\begin{array}{l}\text { Número de pacientes que han } \\
\text { consultado en el último mes al } \\
\text { programa }\end{array}$ & $\begin{array}{l}\text { Número total de pacientes del } \\
\text { programa desde su inicio }\end{array}$ \\
\hline \multirow[t]{7}{*}{ Impacto } & Descompensaciones & $\begin{array}{l}\text { Número de pacientes del } \\
\text { programa que han presentado } \\
\text { descompensación }\end{array}$ & $\begin{array}{l}\text { Número total de pacientes del } \\
\text { programa desde su inicio }\end{array}$ \\
\hline & Altas del programa en metas & $\begin{array}{l}\text { Número de pacientes que regresan } \\
\text { a la red primaria por cumplir } \\
\text { metas de clase funcional, } \\
\text { titulación y educación que } \\
\text { pertenecen al programa }\end{array}$ & $\begin{array}{l}\text { Número total de pacientes del } \\
\text { programa desde su inicio }\end{array}$ \\
\hline & $\begin{array}{l}\text { Reingresos hospitalarios } \\
\text { relacionados con la patología que } \\
\text { controla el programa }\end{array}$ & $\begin{array}{l}\text { Número de pacientes que } \\
\text { re-ingresaron a hospitalización por } \\
\text { la patología que controla el } \\
\text { programa y que pertenecen a este }\end{array}$ & $\begin{array}{l}\text { Número total de pacientes del } \\
\text { programa desde su inicio }\end{array}$ \\
\hline & Consulta por servicio de urgencias & $\begin{array}{l}\text { Número de pacientes que asisten } \\
\text { al servicio de urgencias por } \\
\text { descompensación de patología } \\
\text { que controla el programa }\end{array}$ & $\begin{array}{l}\text { Número total de pacientes del } \\
\text { programa }\end{array}$ \\
\hline & Mortalidad & $\begin{array}{l}\text { Número de pacientes del } \\
\text { programa que mueren }\end{array}$ & $\begin{array}{l}\text { Número total de pacientes del } \\
\text { programa desde su inicio }\end{array}$ \\
\hline & Titulación & $\begin{array}{l}\text { Número de pacientes que } \\
\text { superaron el } 50 \% \text { de la dosis de } \\
\text { betabloqueante que se } \\
\text { encuentran en el programa }\end{array}$ & $\begin{array}{l}\text { Número total de pacientes del } \\
\text { programa desde su inicio }\end{array}$ \\
\hline & Educación & $\begin{array}{l}\text { Número de pacientes que } \\
\text { recibieron al menos el } 50 \% \text { de } \\
\text { sesiones educativas estipuladas en } \\
\text { el programa y que se encuentran } \\
\text { en este }\end{array}$ & $\begin{array}{l}\text { Número total de pacientes del } \\
\text { programa con alteración de la FEV } \\
\text { desde su inicio }\end{array}$ \\
\hline
\end{tabular}


programa multidisciplinar debe atender un mínimo de cincuenta pacientes al mes y contar con:

1. Una enfermera especializada.

2. Un médico Cardiólogo que coordine el manejo de los pacientes y que dedique un $50 \%$ de su tiempo a falla cardiaca o sea especialista en el tema.

3. Un programa educativo para pacientes y cuidadores acerca de insuficiencia cardiaca.

4. Participacioń de los pacientes en el control de los sińtomas y el uso de diuréticos (régimen flexible de diuréticos-peso diario).

5. Seguimiento después del alta y acceso a la atención a través de consultas prioritarias.

6. Programa estructurado de seguimiento programado para titulación de medicamentos.

7. Evaluación de la respuesta al tratamiento (escalas de riesgo).

8. Presentación periódica de indicadores.

9. Juntas médicas para decisiones de manejo avanzado (tabla 2).

\section{Responsabilidad de los aseguradores}

La calidad de la atención no depende exclusivamente de los prestadores de servicios de salud, depende, en gran parte, de los aseguradores que son quienes deben velar y asegurar la máxima calidad de la atención para sus pacientes. Por tanto las EPS deben contar con los siguientes parámetros:

1. Una estrategia de acceso rápido a atención primaria en caso de que el paciente no sea direccionado a una unidad multidisciplinaria después de un egreso por descompensación (plazo máximo de siete días)

2. Tiempo de remisión después de hospitalización al médico especialista o a una unidad multidisciplinar no mayor a quince días.

3. Entrega segura de medicación antes del alta hospitalaria; esta debe estar completa y ajustarse a la formulación emitida por el especialista. Así mismo, dar continuidad a la medicación ambulatoria cumpliendo con los cambios de dosis que genera la titulación.

4. Un plan de autorizaciones expedidas a la IPS que brinda los servicios de Unidad de Falla Cardiaca para consulta prioritaria, consulta de titulación y consultas de educación.

\section{Conclusión}

Como se ha visto hasta ahora, la atención del paciente con falla cardiaca depende de la continuidad de la atención, de la conexión y la buena comunicación de los hospitales con los aseguradores y de estos a la vez con su red de atención primaria $^{8,9}$; así mismo de los pacientes y sus familias quienes deben exigir una atención adecuada y comprometerse con su autocuidado, y finalmente de nosotros como parte de unidades multidisciplinares, de continuar apoyando la formación de médicos, enfermeras y otros profesionales de la salud que intervengan en el cuidado de pacientes con falla cardiaca.

\section{Conflicto de intereses}

Los autores declaran no tener ningún conflicto de intereses.

\section{Bibliografía}

1. McDonagh T, Blue L, Clark A, Dahlstrom U, Ekman I, Lainscak M, et al. European Society of Cardiology Heart Failure Association Standards for delivering heart failure care. Eur J Heart Fail. 2011;13:235-41.

2. Rich MW, Beckham V, Wittenberg C, Leven CL, Freedland KE, Carney RM. A multidisciplinary intervention to prevent the readmission of elderly patients with congestive heart failure. $\mathrm{N}$ Engl J Med. 1995;2:1190-5.

3. ClineCM, Israelsson BYA, Willenheimer RB, Broms K, Erhardt LR. Cost effective management programme for heart failure reduces hospitalisation. Heart. 1998;80:442-6.

4. Jaarsma T, Halfens R, Abu-Saad HH, Dracup K, Gorgels T, van Ree J. Effects of education and support on self-care and resource utilization in patients with heart failure. Eur Heart J. 1999;20:673-82.

5. Stewart S, Pearson S, Horowitz JD. Effects of a home-based intervention among patients with con- gestive heart failure discharged from acute hospital care. Arch Intern Med. 1998;158: 1067-72.

6. Serxner S, Miyaji M, Jeffords J. Congestive heart failure disease management study: a patient education intervention. Prev Manage Congest Heart Fail. 1998;4:23-8.

7. Rich MW, Vinson JM, Sperry JC, Shah AS, Spinner LR, Chung MK, et al. Prevention of readmission in elderly patients with congestive heart failure: results of a prospective, randomized pilot study. J Gen Intern Med. 1993;8:585-90.

8. Gwadry-Sridhar FH, Flintoft V, Lee DS, Lee H, Guyatt GH. A systematic review and meta-analysis of studies comparing readmission rates and mortality rates in patients with heart failure. Arch Intern Med. 2004;164:2315-20, 22.

9. Howlett J, Mann O, Baillie R, Hatheway R, Svendsen A, Benoit R, et al. Heart failure clinics are associated with clinical benefit in both tertiary and community care settings: Data from the Improving Cardiovascular Outcomes in Nova Scotia (ICONS) registry. Can J Cardiol. 2009;25:e306-11. 